\title{
A quasi-dimensional model of turbulence and global charge motion for spark ignition engines with fully variable valvetrains
}

International J of Engine Research 2014, Vol. I5(7) 805-816 (c) IMechE 2014

Reprints and permissions: sagepub.co.uk/journalsPermissions.nav DOI: $10.1177 / 1468087414521615$ jer.sagepub.com (SAGE

\author{
Sebastian Grasreiner', Jens Neumann', Christoph Luttermann', \\ Michael Wensing ${ }^{2}$ and Christian Hasse ${ }^{3}$
}

\begin{abstract}
In this article, a new in-cylinder turbulence modeling approach aims at the improvement of quasi-dimensional simulations for modern spark ignition engines with fully variable valvetrains. Within the derived quasi-dimensional turbulence model, the turbulent production term can physically react on a change of engine operation (e.g. intake valve lift, intake valve timing, engine speed and boost pressure). Moreover, the approach offers access to detailed charge motion quantities for the first time in quasi-dimensional calculations. Hence, it is able to satisfy qualitative and quantitative turbulence descriptions within the entire operating range of the engine.
\end{abstract}

\section{Keywords}

Simulation, spark ignition engines, quasi-dimensional model, turbulence model, charge

Date received: 22 July 2013; accepted: 6 January 2014

\section{Introduction}

Despite purely electric vehicles and alternative concepts, spark ignition (SI) engines still play a key role in globalized individual transportation, which might also be seen as a further trend. The development of efficiencyincreasing engine technologies can be supported by comparably simple and fast quasi-dimensional (QD) simulations of in-cylinder phenomena such as combustion or wall heat transfer. However, as these phenomena are very complex, their simulation demands a highly physical capture of major effects and occurring interactions. In the future, the importance of this aspect even rises as new engine generations are becoming more and more complex due to their increasing number of technical variabilities.

A number of phenomena in the high pressure part of an engine cycle are known to react sensitive to incylinder turbulence. The flame kernel development in the ignition phase and the developing flame spread after inflammation are strongly dependent on turbulence intensity. ${ }^{1}$ Turbulent flame speeds are several times faster than laminar flame propagation, ${ }^{2}$ which has wide-ranging consequences for engine efficiency and emissions. Cyclic variations have their origin in turbulent fluctuations. ${ }^{3-6}$ It is also well known that gasto-wall heat flux depends highly on turbulence. All these effects are of major interest for the simulation of an engine cycle. Hence, the goal is to make turbulence quantities accessible in a QD simulation.

In 1980, Borgnakke et al. ${ }^{7}$ calculated global incylinder turbulence by a $k-\varepsilon$ equation set for QD purposes. The reduction to a simpler one-equation ordinary differential equation (ODE) can be found in the 1988 work of Noske $^{8}$. Later on, Bargende ${ }^{9}$ added a squishing term in 1991 with relevance especially for diesel combustion. Moreover, Bargende introduced a new starting value at intake valve closing (IVC) for the ODE calculation. It empirically takes into account the global velocity, the volumetric efficiency and the intake valve lift. ${ }^{9}$ In 2001, the so far completing step for QD turbulence was established by Pivec ${ }^{10}$ with introduction

\footnotetext{
'BMW Group, München, Germany

${ }^{2}$ Universität Erlangen-Nürnberg, Erlangen, Germany

${ }^{3}$ TU Bergakademie Freiberg, Freiberg, Germany
}

\section{Corresponding author:}

Jens Neumann, BMW Group, 80788 München, Germany.

Email: jens.je.neumann@bmw.de 
Table I. Research engine.

\section{Cylinder/working cycle}

Piston stroke/cylinder bore

Compression ratio/engine displacement

Valves per cylinder

Intake valve actuation (BMW Valvetronic)

Exhaust valve actuation

Load control

Exhaust turbocharger

Fuel supply (BMW High Precision Injection)

Power output (nominal)

Maximum torque
4 in-line $/ 4$ stroke SI

$90.1 \mathrm{~mm} / 84.0 \mathrm{~mm}$

10:1/1996 $\mathrm{cm}^{3}$

2 intake/2 exhaust

Variable timing, continuous variable valve lift and valve phasing (valve lift

difference) at low valve lifts

Variable timing and maximum valve lift

Part load: intake valve lift/timing (throttled operation optional) and near full load: turbocharger with wastegate strategy

Twin scroll concept with optional scavenging operation

Homogeneous, stoichiometric gasoline direct injection, spray-guided operation and multi-hole solenoid valve injector

$180 \mathrm{~kW}$ at $5000-6500 \mathrm{r} / \mathrm{min}$

$350 \mathrm{~N} \cdot \mathrm{m}$ at $1250-4800 \mathrm{r} / \mathrm{min}$

SI: spark ignition.

of a direct injection term. Results of Nefischer et al. ${ }^{11}$ indicate that this developed stage of a turbulence model can still not be fully adopted to the entire operating range of a turbocharged direct injection SI engine with variable intake valve lift. Summarizing, none of these approaches is able to cope with modern complexity of fully variable valvetrains adjustable in intake valve lifts and valve timings because the flow fields and occurring turbulence differ widely. ${ }^{12}$

The newly proposed turbulence model shall physically predict tumble and swirl-based motion and the production of turbulent kinetic energy based on a QD $k-\varepsilon$ approach within all engine operations. It shall be utilized primarily within all types of QD combustion models - most notably the entrainment model ${ }^{13,14}$ and the fractal model ${ }^{15,16}$ - and second within QD wall heat transfer models. Computation time requirements are small compared to three-dimensional (3D) computational fluid dynamics (CFD) simulations. Thus, the long-term vision is QD simulation of in-cylinder phenomena with real-time capability.

In section "Prerequisites," details of the investigated engine and assumptions for the mathematical setting are shown. Section "Governing physics" deduces and summarizes the new approach with key concepts from 3D investigations. Finally, the calibration of the QD model with 3D CFD data and its validation at several significant points of operation are presented in section "Model application, calibration and validation."

\section{Prerequisites}

\section{Research engine details}

In this work, a new generation of SI engines is subject to modeling. The main technologies combined are exhaust gas turbocharging, fully variable valvetrain and gasoline direct injection. The investigated engine represents a four-cylinder derivate of this new engine concept and is used as example for analysis and research (Table 1).

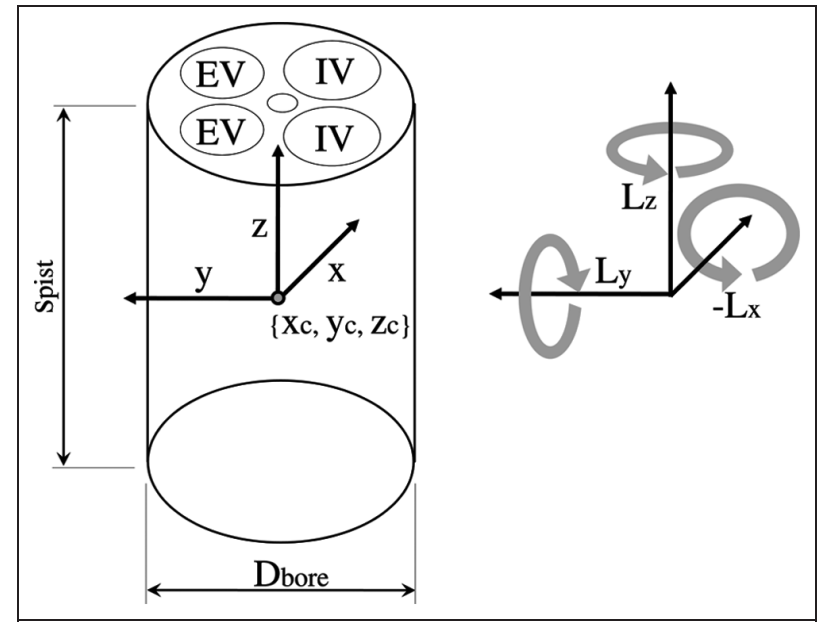

Figure I. Geometry of the simplified combustion chamber (IV, EV) together with orientation of Cartesian coordinates and the coordinate center (left). Global charge motion from IV with main tumble induction $-L_{x}$ and the swirl component $L_{z}$ (right). IV: intake valves; $\mathrm{EV}$ : exhaust valves.

\section{General assumptions for a new modeling approach}

Obviously, the modeling of global flow phenomena without spatial resolution must introduce some simplifications. Therefore, all calculations take place in a strongly simplified combustion chamber with flat cylinder head and piston geometry (Figure 1). In the Cartesian coordinate system, the angular momentum vector $\vec{L}$ describes all occurring motions of charge and can be written in a simplified form as

$$
\vec{L}=\left(\begin{array}{c}
L_{x} \\
L_{y} \\
L_{z}
\end{array}\right)=\left(\begin{array}{c}
J_{x} \cdot \omega_{x} \\
J_{y} \cdot \omega_{y} \\
J_{z} \cdot \omega_{z}
\end{array}\right)=m_{c y l} \cdot\left(\begin{array}{c}
j_{x} \cdot \omega_{x} \\
j_{y} \cdot \omega_{y} \\
j_{z} \cdot \omega_{z}
\end{array}\right)
$$

where $L_{x}$ describes the main tumble component in the cylinder, $L_{y}$ is the minor tumble component and $L_{z}$ can be seen as swirling motion around the cylinder axis. The quantities $J_{i}, j_{i}$ and $\omega_{i}$ represent the global 
momentum of inertia, the mass weighted counterparts and the angular velocity component around the axis $i=\{x, y, z\}$, respectively.

Moreover, some assumptions are introduced in order to derive a new QD production term for turbulent incylinder energy:

- Intake flow is the main driver for charge motion generation. CFD and analytical analyses show a negligible influence of the exhaust flow and blowby flow.

- Air, fuel and residuals in the cylinder are always homogeneous in density and perfectly mixed. Gas viscosity is constant for all temperatures in relevant engine operations.

- Main tumble motion moves around $x$-axis. Minor occurrence of cross- $y$-tumble can be neglected.

- Tumble and swirl motion can be considered independently by superposition.

From a sensitivity analysis of a QD combustion simulation at different engine operation points, it can be shown that the new turbulence model has to provide the turbulent kinetic energy within $\pm 15 \%$ precision to meet a required accuracy of $\pm 5 \%$ for the turbulent flame speed. ${ }^{17}$ A broader confidence interval of $\pm 10 \%$ for the turbulent flame speed would even allow a bandwidth of $\pm 25 \%$ for the turbulent kinetic energy around its effective value. In this context, Schubert ${ }^{18}$ mentions a $30 \%$ deviation for the turbulent kinetic energy as input for convective in-cylinder heat transfer calculations as sufficient.

\section{Governing physics}

\section{Relations between charge motion and turbulence production}

The energy cascade concept describes the energy transfer from large to small scales, which is the driver for turbulence production. The smaller the rotating structures, the higher is the influence of friction effects until the former kinetic energy totally dissipates into internal energy (heat) on Kolmogorov scales. Thus, turbulent fluctuations in an engine cylinder will vanish without continuous energy supply from the mean flow of directed global charge motion.

Modern engine concepts induce either swirl or tumble or a combination of both in the cylinder depending on the valvetrain actuation. So it seems evident to take a closer look at buildup and decay of charge motion ${ }^{19}$ and its interaction with turbulence. This follows the ideas of Poulos and Heywood ${ }^{20}$ taking into account the angular momenta and the rotational energy in every spatial direction. Generally, modeling of turbulence production $d k_{\text {prod }} \propto(\partial U / \partial y)^{2}$ contains the shearing rate of the mean velocity field $U$ of a boundary layer normal to the surface of a flat plate. Ramajo et al. ${ }^{21}$ obtain a modified formulation

$$
d k_{\text {prod }} \propto-\frac{k^{2}}{\varepsilon} \cdot \frac{U^{2}}{l_{t}^{2}}
$$

which can be easily rewritten corresponding to Eiglmeier's approach ${ }^{22}$ using global kinetic energy $E_{r o t}$

$$
d k_{\text {prod }} \propto-\frac{E_{\text {rot }}}{l_{t}} \cdot \sqrt{k}
$$

where $k$ is the QD turbulent kinetic energy, $l_{t}$ is the turbulent length scale and $\varepsilon$ is the dissipation rate. Ramajo et al., ${ }^{21}$ Achuth and Mehta ${ }^{23}$ and Achuth et al. ${ }^{24}$ mention the determination of production terms to be the most significant and hence complex part of QD turbulence modeling. The following sections interpret $d k_{\text {prod }}$ by direct employment of the energy cascade linking turbulence gains directly to losses of the rotational energy $E_{\text {rot }}$

$d k_{\text {prod }} \propto-d E_{\text {rot }}$

In the following, rotational energy $E_{\text {rot }}$ is described as function of angular momenta $L_{d i r}$ because they are seen as global quantities. Recall, local velocity components $U$ of the flow field are not available in a nonspatial approach. The objective is now to find an appropriate closure for $k_{\text {prod }}$, which can cope with different forms of large-scale charge motion and their transfer into small-scale turbulence. Therefore, compression stroke and intake stroke of the engine cycle must be considered independently.

The model of Poulos and Heywood ${ }^{20}$ and enhanced versions ${ }^{25}$ close the production term $k_{\text {prod }}$ by considering a global mean velocity of cylinder charge without depending on a preferred direction. More detailed versions of this closure are derived in the literature by taking into account either only tumble motion $^{21}$ or only swirl motion. ${ }^{22}$ The more general model presented in the following extends these concepts by consideration of both, swirl and tumble.

The following derivation for the production term $k_{\text {prod }}$ is grouped in the phase of closed valves when only shearing influences the production term (section "Turbulence in the compression and expansion stroke (closed valves)") and in the phase of open valves when shearing and additionally the energy of intake valve flow are to be considered (section "Turbulence in the intake stroke (open valves)"). Both shearing and intake effects are again divided into swirl and tumble, respectively. Note that a graphical overview of the model derived in the following is provided in Appendix 1. Table 2 sums up index abbreviations used in the following derivation.

\section{Turbulence in the compression and expansion stroke (closed valves)}

This section derives a term for the rate of change of turbulent kinetic energy $k$ that applies for tumbling and swirl motions at constant cylinder mass. At engine 
Table 2. Frequently used indices.

\begin{tabular}{ll}
\hline Index & \multicolumn{1}{c}{ Description } \\
\hline cyl & In-cylinder-related term \\
dir & Direction of vector decomposition, dir $=\{x y, z\}$ \\
int & Intake stroke-related term \\
IVi & Intake valve numeration, e.g., IVi $=\{I, 2\}$ \\
prod & Production formulation for TKE \\
rot & Rotational energy of the gas mass \\
shr & Shearing stress-related term \\
\hline
\end{tabular}

strokes with closed valves, the term $k_{\text {prod }}$ can only evolve from the existing kinetic energy of the rotation $E_{\text {rot }}$ the cylinder offers. It accumulates shearing effects in directions dir

$$
d k_{\text {prod }}=d k_{\text {prod, shr }}=-\frac{1}{m_{c y l}} \cdot \sum_{d i r} d E_{d i r, r o t, s h r}
$$

Hereby, the rate of change of rotational energy $d E_{\text {dir, rot,shr }}$ is decomposed into $x y$ and $z$ directions by $C_{x y, p r o d} \cdot d E_{x y, r o t, s h r}+C_{z, p r o d} \cdot d E_{z, r o t, s h r} \quad$ representing swirl and tumble motion. The introduced two independent parameters $C_{x y \text {,prod }}$ and $C_{z \text {,prod }}$ are later utilized within the calibration process (see section "QD model calibration").

Subsequently, an energy term is needed for proceeding. The cylinder charge moves with rotational energy

$$
E_{d i r, r o t}=\frac{1}{2} \cdot \frac{L_{d i r}{ }^{2}}{m_{c y l} \cdot j_{d i r}}
$$

Therefore, the change of rotational energy by shearing forces in the gas mass can be easily derived for an assumed quasi-stationary cylinder geometry $\left(d j_{d i r}=0\right)$

$$
d E_{d i r, r o t, s h r}=\frac{1}{2} \cdot \frac{2 \cdot L_{d i r}}{m_{c y l} \cdot j_{d i r}} \cdot d L_{d i r, s h r}=2 \cdot \frac{E_{d i r, r o t}}{L_{d i r}} \cdot d L_{d i r, s h r}
$$

Mass-specific angular inertia $j_{x y}$ of the tumbling gas mass and $j_{z}$ for swirl motion depend on cylinder bore $D_{\text {bore }}$ and the actual piston position $s_{\text {pist }}$ at this time step. The derivation of the terms in equations (8) and (9) is outlined in Appendix 1

$$
\begin{aligned}
& j_{x y}=\frac{1}{4} \cdot\left(\left(\frac{D_{\text {bore }}}{2}\right)^{2}+\frac{s_{\text {pist }}{ }^{2}}{3}\right) \\
& j_{z}=\frac{1}{2} \cdot\left(\frac{D_{\text {bore }}}{2}\right)^{2}
\end{aligned}
$$

Shearing of the charge motion can be used to characterize the production of turbulent kinetic energy ${ }^{22,26}$

$$
\frac{d}{d t} L_{d i r, s h r}=L_{d i r} \cdot \Psi_{d i r} \cdot \sqrt{k}
$$

where $\Psi_{d i r}$ represents the time-decaying function for charge motion (see section "Analysis of time decay for charge motion"). Equation (10) states that current turbulence values have impact on charge motion behavior and thus on later turbulent quantities. Hence, a high turbulent generation early in the engine cycle works against a high turbulence level at combustion.

Together, equations (5)-(10) can reproduce occurring tumble decay, swirl decline and following up turbulence production within the compression stroke.

\section{Turbulence in the intake stroke (open valves)}

The incoming fresh charge during the intake stroke builds up global charge motion and generates intake turbulence directly. This is accounted for in an adapted production term for the intake $d k_{\text {prod, int }}$ added to the established shearing term $d k_{\text {prod, shr }}$

$$
d k_{\text {prod }}=d k_{\text {prod, shr }}+d k_{\text {prod,int }}
$$

In the following, the consideration of contributing flow components (mass flow and gas velocity) over the intake valves $I V i$ is essential. This aims at the derivation of the intake term $d k_{\text {prod,int }}$, which is finished at the end of this section and is fed back into equation (11).

Main tumble $L_{x}$ sets one important type of global charge motion. Tumble production caused by incoming charge within the intake stroke $\left(\dot{m}_{I V i}>0\right)$ writes

$$
\frac{d}{d t} L_{x, I V i, i n t}=j_{x y} \cdot \dot{m}_{I V i} \cdot \mathrm{T} u_{x, I V i} \cdot \omega_{m o t}
$$

where $\mathrm{T} u_{x, I V i}$ is a nondimensional equivalent for tumble generation, based on a steady-state discharge analysis in 3D CFD. The latter refers to a standard methodology in the engine development process for the evaluation of charge motion versus valve lift. It consists of generating an adapted 3D CFD model of the cylinder head, imposing pressure boundary conditions, simulating the flow through the valves at a constant lift, analyzing the mean tumble motion $\mathrm{T} u_{x, I V i}$ at predefined cross sections with a defined algorithm and repeating these steps for different valve lifts. The angular velocity scales with engine speed via $\omega_{\text {mot }}=2 \pi \cdot n_{\text {mot }}$.

Swirl production caused by different incoming mass flows $\left(\dot{m}_{I V i}>0\right)$ over the intake valves $I V i$ depends on differential entry of momentum $\left(\dot{m}_{I V i} \cdot \vec{v}_{I V i}\right)$ and the lever $\vec{r}_{\text {int }}$. Hereby, $\vec{r}_{\text {int }}$ spans from the intake valve center to the $z$-axis within the $x y$-plane of the simplified cylinder roof

$$
\frac{d}{d t} L_{z, I V i, i n t}=\left[\vec{r}_{i n t} \times\left(\dot{m}_{I V i} \cdot \vec{v}_{I V i}\right)\right] \cdot \vec{e}_{z}
$$

Due to valve masking (shadowing of the rear intake valve seat), a high fraction of incoming momentum can be used to generate swirl. Thus, the mainly $y$-directed intake flow allows a simplification of the cross product with $\vec{r}_{z, \text { int }} \perp\left(\dot{m}_{I V i} \cdot \vec{v}_{I V i}\right)$

$$
\frac{d}{d t} L_{z, I V i, \text { int }} \approx r_{z, \text { int }} \cdot \dot{m}_{I V i} \cdot v_{I V i}
$$


Here, $r_{z, \text { int }}$ induces an effective swirl lever (measuring approximately from intake valve center perpendicular to the symmetry plane between both pairs of valves). Note that the components of $\vec{r}_{\text {int }}$ and $r_{z, \text { int }}$ have different signs for various intake valves.

If mass flow exits the cylinder through valves $I V i$ $\left(\dot{m}_{I V i}<0\right)$, the loss of angular momentum fraction writes

$$
\frac{d}{d t} L_{d i r, I V i, i n t}=L_{d i r} \cdot \frac{\dot{m}_{I V i}}{m_{c y l}}
$$

Overall angular momentum change for different valves $I V i$ combines as

$$
d L_{d i r, \text { int }}=\sum_{I V i} d L_{d i r, I V i, \text { int }}
$$

This sum is valid for every two scalar component directions $x y$ and $z$ independently and contains the signs of production " + " and destruction "-".

Total tumble change at the intake stroke consists of shearing decay (destruction of angular momentum) and kinetic buildup for all intake valves $I V i$

$$
d L_{x y}=d L_{x y, s h r}+d L_{x y, \text { int }} \approx d L_{x y, s h r}-d L_{x, \text { int }}
$$

The second part of equation (17) expresses the length of the vector $\vec{L}_{x y}$ by major and minor tumble components $L_{x}$ and $L_{y}$ via superposition $\sqrt{ } L_{x}^{2}+L_{y}^{2}$ combined with the assumption of negligible crosstumble $L_{x}^{2} \gg L_{y}^{2}$ and via correction of the algebraic sign (compare $-L_{x}$ in Figure 1).

Overall swirl change at the intake stroke consisting of shearing and a kinetic balance for all intake valves $I V i$ is written as

$$
d L_{z}=d L_{z, s h r}+d L_{z, \text { int }}
$$

The buildup of rotational energy $E_{\text {rot, int }}$ within the cylinder is fed from all charge motion in directions dir similar to equation (7)

$$
d E_{r o t, \text { int }}=2 \cdot \sum_{d i r}\left(\frac{E_{d i r}, r o t}{L_{d i r}} \cdot d L_{d i r, \text { int }}\right)
$$

Mean flow kinetic energy $\mathrm{KE}_{\text {int }}$ from the entering gas mass $\left(\dot{m}_{I V i}>0\right)$ for all intake valves $I V i$ balances

$$
d \mathrm{KE}_{i n t}=\frac{1}{2} \cdot \sum_{I V i}\left(\dot{m}_{I V i} \cdot\left(\vec{v}_{I V i}\right)^{2}\right) d t
$$

Intake production considers how much of induced kinetic energy produces "instantaneous" turbulence. Thus, the difference between intake mean flow kinetic energy $\mathrm{KE}_{\text {int }}$ and global charge motion buildup $E_{r o t, \text { int }}$ is a measure for instant shearing turbulence. $C_{\text {prod, int }}$ in equation (21) works as proportional factor and accounts for the turbulent efficiency at high dissipation rates for the intake flow

$$
d k_{\text {prod, int }}=C_{\text {prod, int }} \cdot \frac{d \mathrm{KE}_{\text {int }}-d E_{\text {rot, int }}}{m_{c y l}}
$$

Finally, the general turbulence production term for the intake stroke (equation ((11))) adds up shearing $d k_{\text {prod, shr }}$ and intake buildup $d k_{\text {prod, int }}$.

\section{Analysis of time decay for charge motion}

As mentioned previously, charge motion and especially the tumble motion decays during compression. This decay is known to highly depend on the piston position and may be expressed as a function $\Psi$. In this section, the piston position dependence (geometric dependency) of $\Psi$ is investigated considering its analytical background and using a simplified test case in 3D CFD.

For the integration of the general shearing equation (10), it is assumed that $\Psi$ and $k$ are independent of time (quasi-stationary) yielding

$$
L(t)=e^{\Psi \cdot \sqrt{k} \cdot t}
$$

Hence, for the initialized state $\left\{L_{0}, k_{0}\right\}$ at the starting time $t_{0}=0$, the decay function can be defined as gradient like

$$
\Psi=\left.\frac{1}{\sqrt{k_{0}}} \cdot \frac{d}{d t}\left(\frac{L}{L_{0}}\right)\right|_{t_{0}}
$$

Equation (23) only considers shearing influences on angular momentum decay. Therefore, geometric boundaries such as the moving piston and valves are frozen for this ODE initial value problem. The unit of the decay function $\Psi$ is expected to act like an inverse length scale $(1 / \mathrm{m})$.

In order to determine the general shape of the decay function in equation (23), the following idealized setup is investigated using $3 \mathrm{D}$ CFD by

1. Definition of the static cylinder geometry (piston position at a certain crank angle (CA)),

2. Employment of the initial state $\left\{L_{0}, k_{0}\right\}$ for the whole cylinder gas mass,

3. Analysis of charge motion decay versus time.

For the initial state in the cylinder, a 3D CFD geometry of the investigated engine with all valves closed in the compression stroke is used. The general aim is to keep angular momentum of the charge motion constant for different piston positions. Its mathematical form with the assumption of homogeneous density $\rho$ is written as

$$
\vec{L}=\rho \cdot \int_{V_{c y l}} \vec{r} \times \vec{v} d V
$$

Here, the cross product of velocity field $\vec{v}$ and effective lever $\vec{r}$ is integrated into the cylinder volume $V_{c y l}$. For the main tumble component, vector decomposition yields

$$
L_{x} \propto \int_{V_{c y l}}\left(r_{y} \cdot v_{z}-r_{z} \cdot v_{y}\right) d V \rightarrow \text { const }
$$




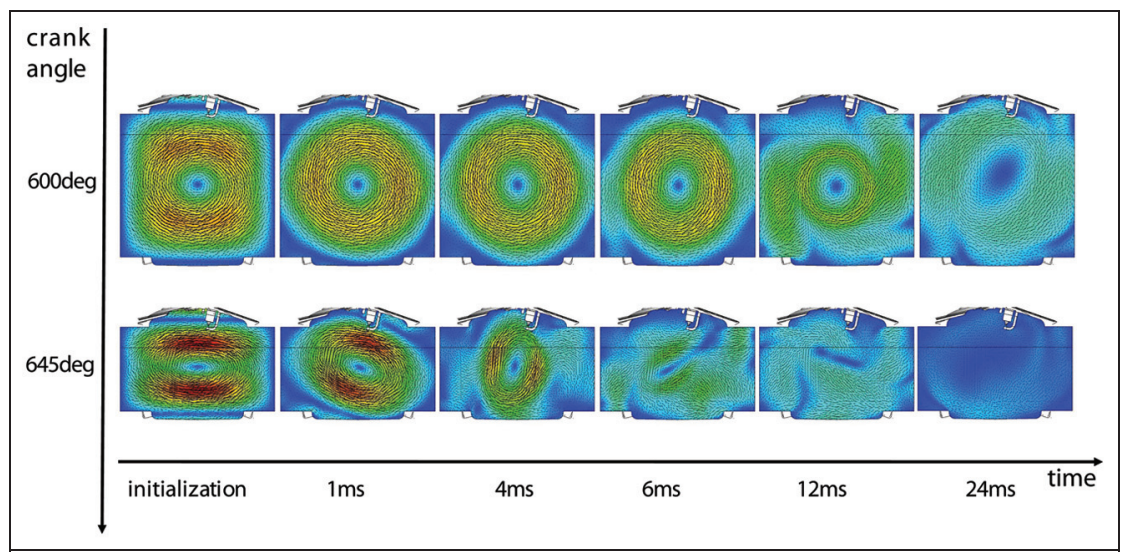

Figure 2. Static tumble decay of the 3D Taylor-Green initialization. Cross section of the $y z$ plane. Sample crank shaft positions at $600^{\circ} \mathrm{CA}$ with $s_{\text {pist }} / D_{\text {bore }}=I$ and at $645^{\circ} \mathrm{CA}$ with $s_{\text {pist }} / D_{\text {bore }}=0.61$. Velocity flow fields are equally scaled.

Thus, the $x$-tumble depends on the levers $r_{y}$ and $r_{z}$ as well as on the velocity components $v_{z}$ and $v_{y}$. The lever in $y$ direction is fixed with cylinder bore. In $z$ direction, there occurs a permanent change of the lever length in the working cycle according to the actual piston position, which then is written as

$$
\begin{gathered}
r_{y} \propto D_{\text {bore }}=\mathrm{const} \\
r_{z} \propto s_{\text {pist }}
\end{gathered}
$$

For the initial flow field, this demands to adapt the velocity component $v_{y}$ to constantly fulfill relation (25). The velocity vectors were initialized according to a simplified two-dimensional (2D) Taylor-Green vortex form like

$$
\vec{v}=\left(\begin{array}{c}
v_{x} \\
v_{y} \\
v_{z}
\end{array}\right)=\left(\begin{array}{c}
0 \\
\Gamma \cdot \cos \left(y \cdot \pi / D_{\text {bore }}\right) \cdot \sin \left(z \cdot 2 \pi / s_{\text {pist }}\right) \\
\sin \left(y \cdot 2 \pi / D_{\text {bore }}\right) \cdot \cos \left(z \cdot \pi / s_{\text {pist }}\right)
\end{array}\right)
$$

In equation (27), $\Gamma$ represents an artificial amplification of the original velocity component $v_{y}$. Here, $\Gamma \propto\left(D_{\text {bore }} / s_{\text {pist }}\right)$ is chosen iteratively to obtain a constant main tumble component initialization $L_{x}$ for piston positions near bottom dead center (BDC) and top dead center (TDC) as well.

For the starting value of turbulent state $k_{0}$ in equation (23), mean velocity field $v$ and velocity fluctuation $v^{\prime}$ were scaled in a simple isotropic estimation

$$
v^{\prime}=\sqrt{\frac{2}{3}} k \approx 2 \% \cdot v
$$

Here, the mean velocity $v$ was defined to be of a typical value of $25 \mathrm{~m} / \mathrm{s}$ at BDC. Using the obtained definition of tumble initialization, the procedure was repeated for different piston positions in the real cylinder geometry and the decay behavior was investigated.

Figure 2 illustrates how the induced tumble $L_{x}$ alters versus time. After initialization, the vortex rapidly decays especially at the cylinder boundaries. The tumble cannot preserve its original shape because friction effectively causes its deformation. Moreover, it becomes evident that charge motion decay is strongly linked to geometric boundary conditions. The further a rotating motion is stretched or compressed, which means $s_{\text {pist }} / D_{\text {bore }} \neq 1$ in this manner, the faster the energy transfer away from large scales is. The measured time scales for reducing the tumble motion by a factor of 2 are therefore $24-25 \mathrm{~ms}$ at $600^{\circ} \mathrm{CA}$ and only 6-7 ms at $645^{\circ} \mathrm{CA}$. The reason for this can be seen in gradually higher mean stress rates for the flow field at piston positions located nearer to TDC (Figure 3). The mean stress rates drive the charge motion decay. The more the piston evolves to TDC, the faster the kinetic energy transfers into small-scale turbulence, which can be seen from the earlier turbulence peaks in Figure 3 on the right.

The whole procedure of tumble decay observation can be transferred to swirl analysis with only the adaption of the Taylor-Green initialization. Therefore, the velocity profiles have to be turned and the angular momentum conservation is applied again

$$
L_{z} \propto \int_{V_{c y l}}\left(r_{x} \cdot v_{y}-r_{y} \cdot v_{x}\right) d V \rightarrow \text { const }
$$

together with fixed cylinder boundaries $r_{x}, r_{y} \propto D_{\text {bore }}=$ const.

Figure 4 shows the different decay functions for tumbling gas motion (around $x$ - and $y$-axes with the assumption of rotational symmetry $\Psi_{x}=\Psi_{y}=: \Psi_{x y}$ ) and swirl motion (around $z$-axis). Whereas the tumble decay has an optimum with least tumble destruction per time step, the swirl decline is monotonic along the piston movement from BDC to TDC. The reason is obvious: tumbling motion is rapidly deformed because it has its rotational axis perpendicular to the piston translation. Thus, the degree of geometric disturbance defines the gradient of tumble decay. The monotonic swirl decline function constitutes that the swirl motion is only compressed. Its frictional forces are increasing during compression, but the swirl vortex can preserve its shape. Thus, the swirl motion lasts longer than the 


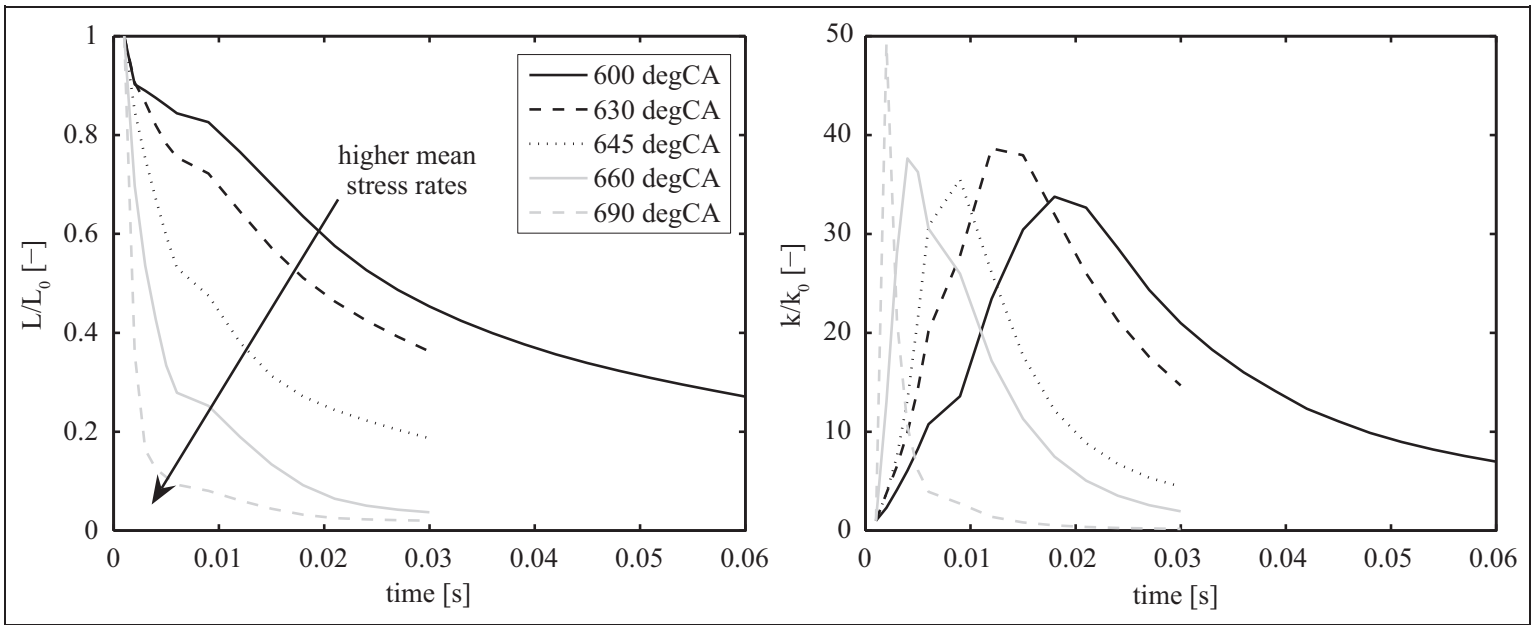

Figure 3. Static tumble decay versus time after 3D Taylor-Green initialization. Normalized global charge motion for different crank shaft positions (left) and according to normalized turbulence development (right). Subscript "0" refers to the initialization state at $t_{0}=0$.

CA: crank angle.

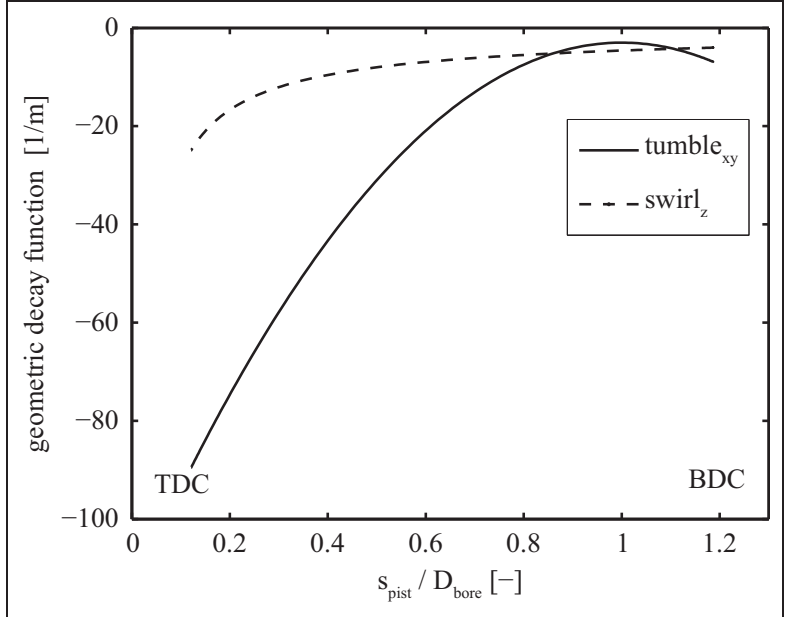

Figure 4. Decay functions for different types of charge motion derived from 3D Taylor-Green experiments without piston motion.

TDC: top dead center; BDC: bottom dead center.

tumble. As a consequence, swirl and tumble differ in their contribution to turbulence production. Again, it is important to analyze them independently with later superposition. In the following, the acquired decay functions are utilized in the process of model calibration and validation for the evaluation of equation (10) by means of interpolation in tabulated data.

\section{Model application, calibration and validation}

The QD turbulence model derived in section "Governing physics" is embedded in a one-equation ODE for global in-cylinder turbulent kinetic energy (compare Appendix 1). Subsequently, it is evaluated for different engine operations, offering an insight into the model's general behavior and tendencies.

\section{QD model application}

Figure 5 shows the function for a sample engine cycle at $2000 \mathrm{r} / \mathrm{min}$ and $2 \mathrm{~mm}$ mean intake valve lift. Due to intake phasing (valve lift curves differ), a differing mass flow $d m_{I V i} / d t$ enters for each intake valve (Figure 5(a)). Hence, there is not only a tumble motion $L_{x y}$ created but also a swirl component $L_{z}$ in the cylinder (Figure 5(b)). In fact, the swirl component even dominates this engine operation, because an early valve closing at the intake inhibits further tumble buildup.

The reaction of turbulence can be seen in Figure 5(c) and (d). As expected, there is a high peak at the very beginning of the cycle, caused by high intake velocities. This peak is enhanced by a short fuel direct injection followed by a significant gradient of turbulence destruction. Nearly at ignition TDC $\left(720^{\circ} \mathrm{CA}\right)$, there occurs a secondary turbulence maximum for this cycle. Here, the swirl motion transfers its long-lasting energy to produce small-scale turbulence, which will later dissipate after ignition top dead center (ITDC).

\section{QD model calibration}

As the introduced turbulence model was constructed upon a physical basis, an adjustment of the production terms for $k_{\text {prod }}$ is possible with minimum effort. The model calibration was performed at an engine operation with $2000 \mathrm{r} / \mathrm{min}$, bmep 2 bar because this offers the occurrence of tumble and swirl motion at the same time.

The used optimizing algorithm is based on a gradient determination for least-square error minimization and coupled with the MATLAB solver for the described 


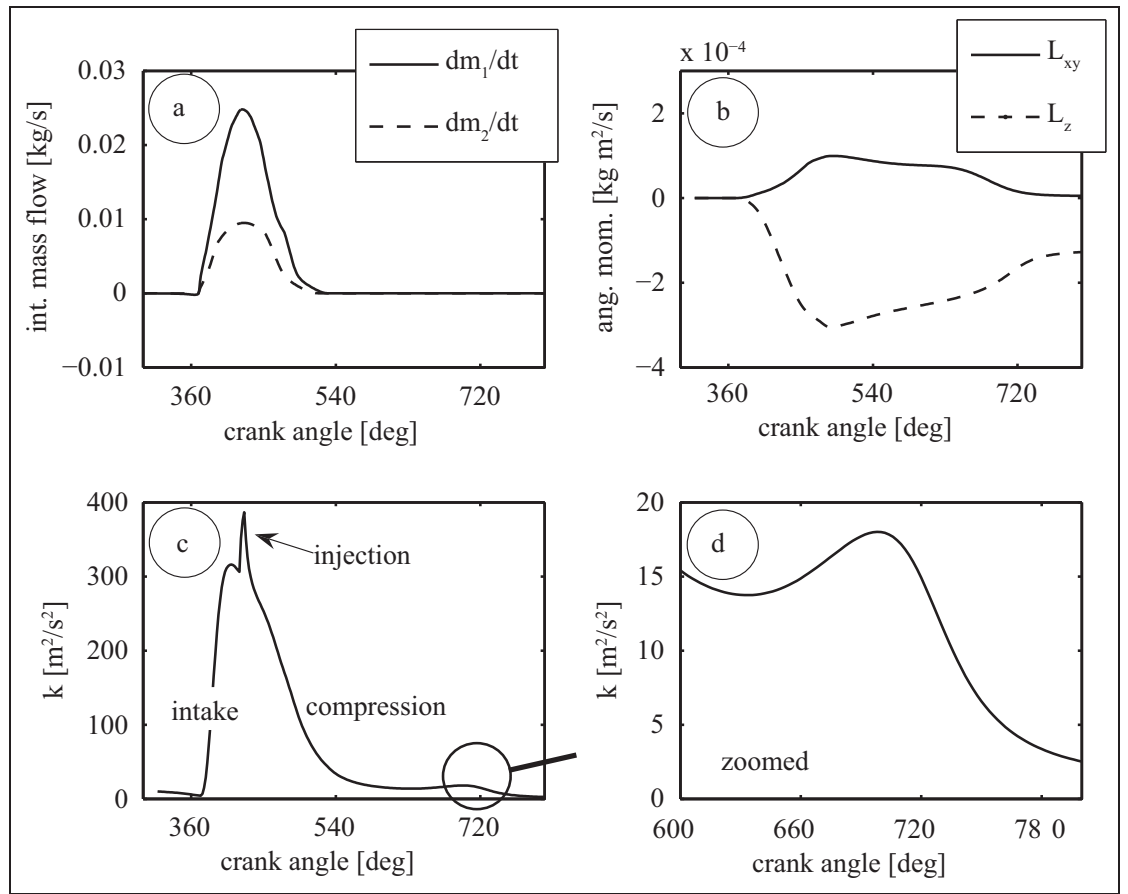

Figure 5. Application of the QD approach at sample operation with $2000 \mathrm{r} / \mathrm{min}, 2 \mathrm{~mm}$ valve lift and early IVC: (a) incoming mass flows over phased intake valves and (b) thus the buildup of charge motion potential causes (c, d) turbulent buildup within the compression stroke.

equation set. The algorithm was given a criterion to match the characteristics of turbulence near ITDC (relative to 3D CFD reference solutions described in the following). This criterion involves the weighted error sum of $k$ at IVC, $k$ at the combustion peak and the CA of this secondary maximum of $k$ close to TDC. The turbulence production terms from instantaneous intake turbulence, swirl decline and tumble decay were optimized only with the help of the variable parameters $C_{\text {prod,int }}$ (equation (21)), $C_{z \text {,prod }}$ and $C_{x y, \text { prod }}$ (implemented as sub-parameters in sum of equation (5) for each direction) resulting in the following causality for the parameters

$$
0.05 \approx C_{\text {prod, int }} \ll C_{z, \text { prod }}<C_{x y, \text { prod }}<1
$$

Beside the production term, a concurrent standard calibration of the entire one-equation turbulence model (see Appendix 1) was performed. Hereby, only the terms with the contribution for density $d k_{d e n s}$ and for dissipation $\varepsilon \cdot d t$ were considered and incorporated into the optimization procedure described above by addition of corresponding calibration parameters. The total number sums up to five calibration parameters, compared with mostly less parameters for other, less detailed turbulence models. All found calibration parameters for the turbulence model were later applied unchanged to the upcoming comparisons.

\section{QD model validation and proof of concept}

As not only engine speed but also intake valve lift and timing have a great impact on turbulence and global charge motion, validation points were selected in a way that all these features vary (Table 3 ).

In order to verify the functionality of the introduced QD turbulence model, results from 3D CFD are created for every validation point. This data basis provides the different types of charge motion, the turbulent state, turbulence dissipation and turbulent length scale as a function of CA within the cycle. For this purpose, adapted experiments of the dynamic gas exchange analysis are conducted in 3D CFD using ANSYS CFX. This involves to start the computation at intake valve opening (IVO), to neglect the combustion ("cold procedure" ${ }^{27}$ ) and to end the computation after ITDC. As typical in-cylinder measurements provide flow field quantities and thus only indirect turbulence information, the described CFD procedure involves less efforts and more precise reference data compared with measurements.

Here, it is important to note that the $3 \mathrm{D}$ intake flow shows a high complexity and is widely anisotropic. Thus, it is neither expected nor important to rebuild the occurring local intake phenomena by a QD modeling approach. So, the following validation is concentrated on the comparison of all quantities after the closure of the intake valves. Nevertheless, QD equations (11)-(21) are applied for the whole duration of the opened intake valves. The required air mass flows through the opened intake valves are provided by a prior gas exchange analysis for the evaluation of charge motion buildup. The error of the total inducted mass compared with the CFD reference solution does not exceed $2 \%$.

In Figure 6, validation results are shown for the engine's operation point swirl 1. Tumble component 
Table 3. Operation points for QD model validation.

\begin{tabular}{lllrrrr}
\hline Operation state & $\begin{array}{c}\text { Engine speed } \\
(\mathrm{r} / \mathrm{min})\end{array}$ & IV close timing & IV lift & $\begin{array}{c}\text { Manifold pressure } \\
(\mathrm{mbar})\end{array}$ & Relative swirl & Relative tumble \\
\hline swirl I & 2000 & $530^{\circ} \mathrm{CA}$ & $2 \mathrm{~mm}$, phasing & 950 & 1 & 0.1 \\
swirl 2 & 2000 & $560^{\circ} \mathrm{CA}$ & $4 \mathrm{~mm}$, phasing & 950 & 0.25 & 0.25 \\
tumble 1 & 2000 & $630^{\circ} \mathrm{CA}$ & $10 \mathrm{~mm}$ & 950 & 0 & 0.25 \\
tumble 2 & 6000 & $610^{\circ} \mathrm{CA}$ & $10 \mathrm{~mm}$ & 2000 & 0 & 1 \\
\hline
\end{tabular}

IV: intake valve; CA: crank angle.

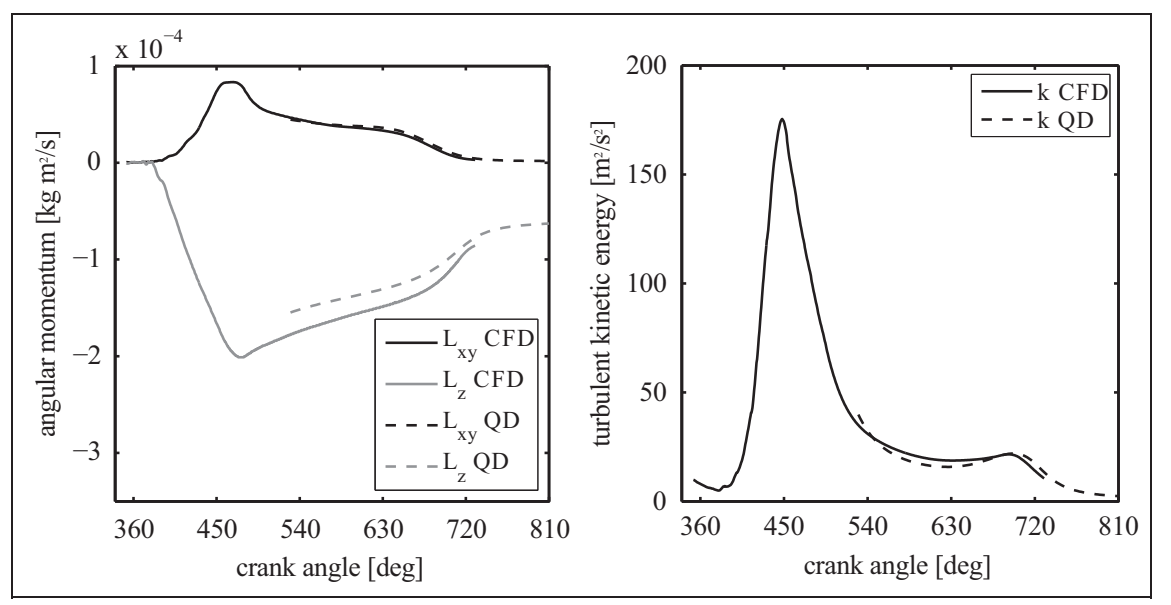

Figure 6. Validation of charge motion and turbulence at operation state swirl I. CFD: computational fluid dynamics; QD: quasi-dimensional.

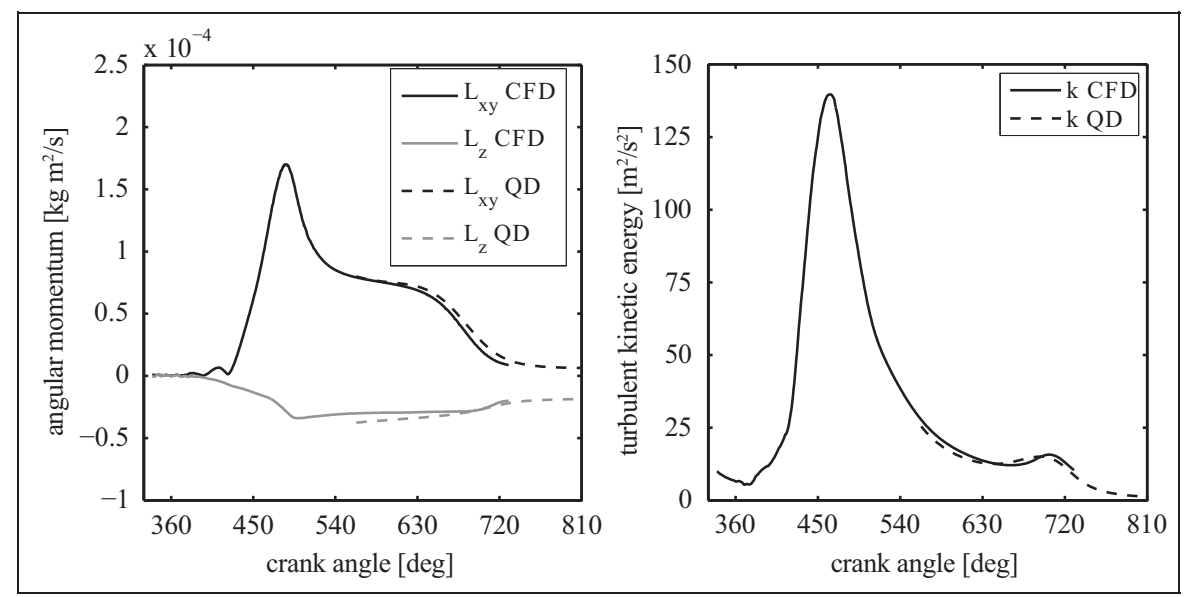

Figure 7. Validation of charge motion and turbulence at operation state swirl 2.

CFD: computational fluid dynamics; QD: quasi-dimensional.

$L_{x y}$ is matched well between QD approach and 3D CFD. The swirl motion $L_{z}$ is slightly underestimated. Nevertheless, the QD turbulence quality $k$ is predicted very well and lies within a $\pm 10 \%$ accuracy at $690^{\circ} \mathrm{CA}$ (i.e. at a crank position close to ignition).

For the operation at swirl 2, the swirl component $L_{z}$ is less dominant compared to the tumble component $L_{x y}$ (Figure 7 ). Both charge motions are predicted very well at IVC (beginning of the dashed lines) in quantity and general quality by the QD approach. Thus, the turbulence quality $k$ also matches very well and lies within an accuracy of $\pm 15 \%$ at $690^{\circ} \mathrm{CA}$.

Figure 8 shows the comparison between QD and 3D CFD results for the engine operation at tumble 1 . It must be mentioned here that for tumble operation, the swirl component $L_{z}$ at IVC should vanish in 3D CFD 


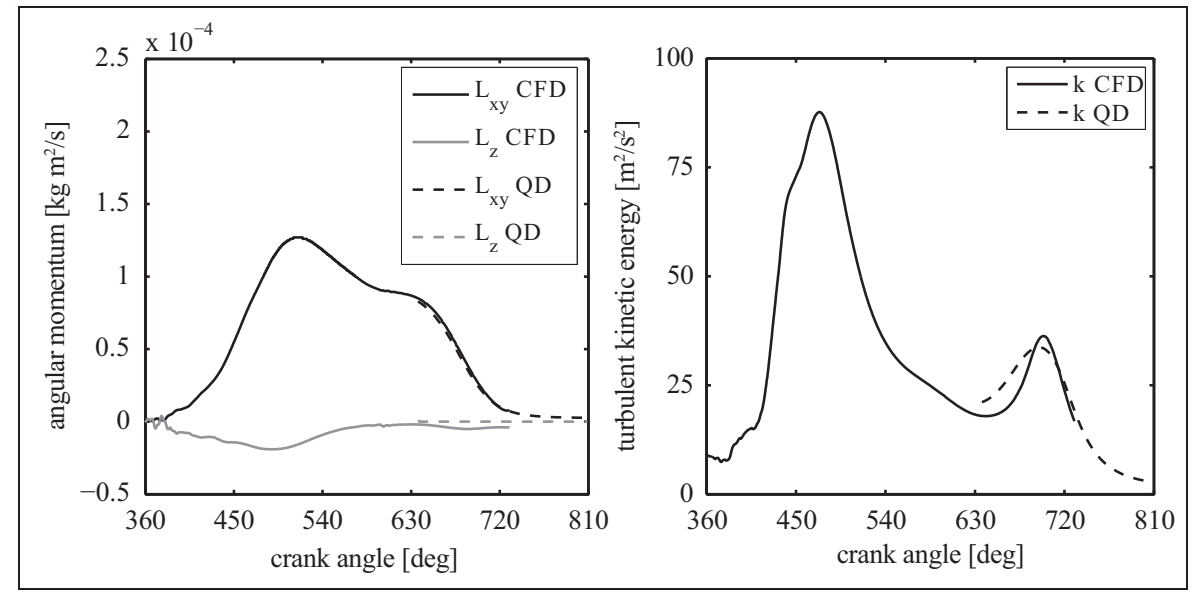

Figure 8. Validation of charge motion and turbulence at operation state tumble $I$. CFD: computational fluid dynamics; QD: quasi-dimensional.

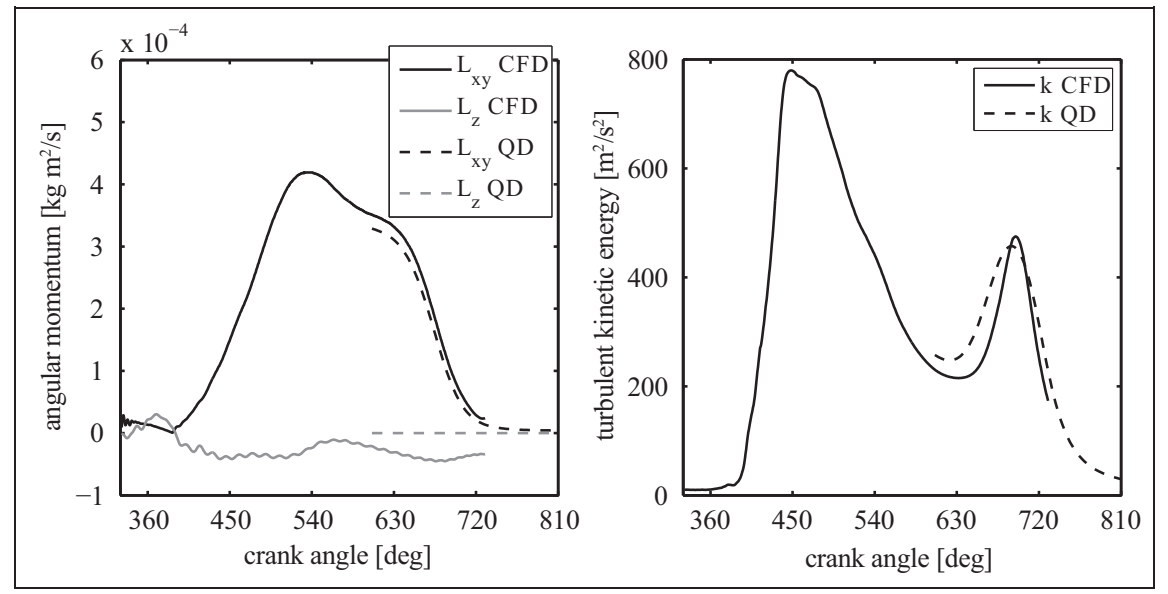

Figure 9. Validation of charge motion and turbulence at operation state tumble 2.

CFD: computational fluid dynamics; QD: quasi-dimensional.

because both intake valves let enter the same air mass flow. The remaining $L_{z}$ values in the $3 \mathrm{D}$ results evolve from in-cylinder flow field irregularities that are not implemented in the QD equations. But compared with the amplitude of the tumble $L_{x y}$ (10 times higher at IVC), these effects can be neglected. The tumble buildup and decay are predicted very well by the QD approach. QD turbulent kinetic energy $k$ shows an earlier peak for QD compared to 3D CFD. Its accuracy still remains within $\pm 15 \%$ at $690^{\circ} \mathrm{CA}$.

Figure 9 illustrates the validation for the high load and high engine speed operation tumble 2. The predominant tumble motion $L_{x y}$ is predicted very well in decay quality. Due to the high engine speed, the turbulence level $k$ is much higher compared to the other validation points. Although its secondary peak occurs around $5^{\circ} \mathrm{CA}$ too early, the new QD approach predicts the turbulence at $690^{\circ} \mathrm{CA}$ within a good accuracy of $< \pm 10 \%$ compared to $3 \mathrm{D}$ results. Again, the negligible swirl component $L_{z}$, which exists in 3D CFD, cannot be reproduced by the QD model. In summary, the produced model results lie within the expected confidence interval (especially at $690^{\circ} \mathrm{CA}$ ) and generally show a good quality.

\section{Conclusion}

In order to reduce fuel consumption, new SI engines adopt an increasing number of variabilities. This makes their operation and the predictive simulation of their operation a challenging task. As a contribution, this work presents a new QD model for the prediction of incylinder charge motion and turbulence. It aims for the application to engines with fully variable valvetrains. Due to its physical background, it can be applied within the QD simulation of in-cylinder phenomena offering the long-term vision of real-time capability. The model is calibrated and thoroughly validated by means of $3 \mathrm{D}$ 
CFD simulations on a state-of-the-art research engine. Global charge motion and turbulence can be predicted within an envisaged confidence interval at operating points with wide variation of actuation. This allows the application of the model in the context of QD simulation of combustion and heat transfer.

\section{Acknowledgements}

The authors thank B. Durst, B. Hussmann, A. Kleemann, U. Knoll, D. Linse, A. Nefischer and L. Schaefer of BMW Group for their support.

\section{Declaration of conflicting interests}

The authors declare that there is no conflict of interest.

\section{Funding}

This research received no specific grant from any funding agency in the public, commercial or not-for-profit sectors.

\section{References}

1. Ewald J. A level set based flamelet model for the prediction of combustion in homogeneous charge and direct injection spark ignition engines. PhD Thesis, RWTH Aachen, Aachen, 2006.

2. Peters N. Technische Verbrennung. Aachen: ITV, RWTH, 2009.

3. Hasse C, Sohm V and Durst B. Numerical investigation of cyclic variations in gasoline engines using a hybrid URANS/LES modeling approach. Comput Fluids 2010; 39: 25-48.

4. Goryntsev D, Sadiki A, Klein M and Janicka J. Large eddy simulation based analysis of the effects of cycle-tocycle variations on air-fuel mixing in realistic DISI ICengines. P Combust Inst 2009; 32: 2759-2766.

5. Pischinger R, Kell M and Sams T. Thermodynamik der Verbrennungskraftmaschine. Wien: Springer-Verlag, 2009.

6. Zigan L, Shi JM, Krotow I, Schmitz I, Wensing M and Leipertz A. Fuel property and fuel temperature effects on internal nozzle flow, atomization and cyclic spray fluctuations of a direct injection spark ignition injector. Int J Engine Res 2013; 14(6): 543-556.

7. Borgnakke C, Arpaci V and Tabaczynski R. A model for the instantaneous heat transfer and turbulence in a spark ignition engine. SAE paper 800287, 1980.

8. Noske G. Ein quasidimensionales Modell zur Beschreibung des ottomotorischen Verbrennungsablaufs. Düsseldorf: VDI Fortschrittsberichte Nr. 6(211), 1988.

9. Bargende M. Ein Gleichungsansatz zur Berechnung der instationären Wandwärmeverluste im Hochdruckteil von Ottomotoren. PhD Thesis, TU Darmstadt, Darmstadt, 1991.
10. Pivec R. Quasidimensionale Modellierung des gasseitigen Wärmeüberganges in Verbrennungsmotoren. PhD Thesis, Technische Universität Graz, Graz, 2001.

11. Nefischer A, Neumann J, Stanciu A and Wimmer A. Comparison and application of different phenomenological combustion models for turbo-charged SI engines. FISITA paper 2010-C-170, 2010.

12. Bossung $\mathrm{C}$ and Bargende M. Turbulenzmodellierung. Frankfurt: Research project Nr 1067, Forschungsvereinigung Verbrennungskraftmaschinen e.V., 2010.

13. Blizard NC and Keck JC. Experimental and theoretical investigation of turbulent burning model for internal combustion engines. SAE paper 740191, 1974.

14. Tabaczynski RJ, Ferguson CR and Radhakrishnan K. A turbulent entrainment model for spark-ignition engine combustion. SAE paper 770647, 1977.

15. Santavicca DA, Liou D and North GL. A fractal model of turbulent flame kernel growth. SAE paper 900024, 1990.

16. D'Errico G and Onorati A. Thermo-fluid dynamic modelling of a six-cylinder SI engine with a secondary air injection system. Int J Engine Res 2006; 7(1): 1-16.

17. Grasreiner S. Combustion modeling for virtual SI engine calibration with the help of $0 D / 3 D$ methods. PhD Thesis, TU Bergakademie Freiberg, Freiberg, 2012.

18. Schubert C. Drall- und Tumblemodellierung zur Berechnung des Wandwärmeüberganges. Master Thesis, Technische Universität Graz, Graz, 2001.

19. Dai W, Newman CE and Davis GC. Predictions of in-cylinder tumble flow and combustion in SI engines with a quasi-dimensional model. SAE paper 961962, 1996.

20. Poulos SG and Heywood JB. The effect of chamber geometry on spark ignition engine combustion. SAE paper 830334, 1983.

21. Ramajo DE, Zanotti AL and Nigro NM. Validación de un modelo de tumble y turbulencia para un motor de cuatro válvulas de alto rendimiento. Mecánica Comput 2005; 24(8): 1527-1542.

22. Eiglmeier C. Phänomenologische Modellbildung des gasseitigen Wandwärmeüberganges in Dieselmotoren. $\mathrm{PhD}$ Thesis, Universität Hannover, Hannover, 2000.

23. Achuth M and Mehta PS. Predictions of tumble and turbulence in four valve pentroof spark ignition engines. Int J Engine Res 2001; 2(3): 209-228.

24. Achuth M, Subramanian G and Mehta PS. Model predictions for tumble and turbulence in SI engine combustion chambers. SAE paper 2001-28-0013, 2001.

25. Bozza F, Gimelli A, Merola S and Vaglieco B. Validation of a fractal combustion model through flame imaging. SAE paper 2005-01-1120, 2005.

26. Ramos JI. Internal combustion engine modeling. New York: Hemisphere Publishing Corp., 1989.

27. Linse D, Hasse C and Durst B. An experimental and numerical investigation of turbulent flame-propagation and flame structure in a turbo-charged direct injection gasoline engine. Combust Theor Model 2009; 13: 167-188. 


\section{Appendix I}

\section{Summary for the quasi-dimensional turbulence model}

Table 4. Key relations for the new QD turbulence model.

$$
\begin{gathered}
\text { One-equation } k-\varepsilon \text { turbulence balance } \\
d k=d k_{\text {prod }}+\left(d k_{\text {int }}+d k_{\text {exh }}+d k_{\text {inj }}\right)+d k_{\text {dens }}+d k_{\text {squish }}-\varepsilon \cdot d t \\
\text { Overall turbulent production prod } \\
d k_{\text {prod }}=d k_{\text {prod, shr }}+d k_{\text {prod,int }}
\end{gathered}
$$

Shearing production shr (all engine strokes) $d k_{\text {prod, shr }} \propto-d E_{\text {rot, shr }}$

\section{Decay of}

rotational energy rot

$d E_{\text {rot, shr }} \propto \sum_{\text {dir }} d L_{\text {shr }}$

\section{Tumble decay} and swirl decay dir $\frac{d}{d t} L_{\text {dir }, \text { shr }}=L_{\text {dir }} \cdot \Psi_{\text {dir }} \cdot \sqrt{k}$

Decay function $\Psi=f\left(s_{\text {pist }} / D_{\text {bore }}\right)$

$$
\begin{aligned}
& \text { Instantaneous intake turbulence int } \\
& \text { (intake stroke, intake valves open) } \\
& d k_{\text {prod, int }} \propto\left(d K E_{\text {int }}-d E_{\text {rot, int }}\right)
\end{aligned}
$$

$$
\begin{gathered}
\text { Intake mean flow kinetic energy } \\
\frac{d}{d t} K E_{\text {int }}=1 / 2 \cdot \sum_{I V_{i}}\left(\dot{m}_{I V i} \cdot\left(\vec{\nu}_{I V i}\right)^{2}\right) \\
\text { Buildup of } \\
\text { rotational energy rot } \\
d E_{\text {rot, int }} \propto \sum_{\text {dir }} d L_{\text {int }}
\end{gathered}
$$

Tumble production $x$

$\frac{d}{d t} L_{x, i n t} \propto \sum_{I V i} \dot{m}_{I V_{i}} \cdot T u_{x, I V i}$

Swirl production z $\frac{d}{d t} L_{z, i n t} \propto \sum_{I V i} \dot{p}_{I V i}$

Tumble generation

Momentum flow $\dot{p}_{I V i}=\dot{m}_{I V_{i}} \cdot \nu_{I V_{i}}$

exh: exhaust-related term; inj: injection-related term; dens: density variation-related term, squish: squish flow-related term; $L_{V V}:$ valve lift.

\section{Quasi-dimensional derivation of the mass moment of inertia}

Deriving the moment of inertia $j_{d i r}$ for all directions $d i r$ is required for the rotational energy $E_{d i r}$,rot . Here, some geometric correlations are needed. The simplified pancake cylinder geometry is bounded by its bore diameter $D_{\text {bore }}$ and the actual piston stroke $s_{\text {pist }}$ (Figure 1). The rotating charge motion is assumed to be centered at the coordinates $\left\{x_{c}, y_{c}, z_{c}\right\}$. Formulations for mass moments of inertia can be taken from engineering mechanics textbooks

$$
\begin{aligned}
& J_{x}=\int_{m_{c y l}}\left(\left(y-y_{c}\right)^{2}+\left(z-z_{c}\right)^{2}\right) d m_{c y l} \\
& J_{y}=\int_{m_{c y l}}\left(\left(x-x_{c}\right)^{2}+\left(z-z_{c}\right)^{2}\right) d m_{c y l} \\
& J_{z}=\int_{m_{c y l}}\left(\left(x-x_{c}\right)^{2}+\left(y-y_{c}\right)^{2}\right) d m_{c y l}
\end{aligned}
$$

The following mathematical ideas can be applied:

- $\quad d m_{c y l}=\rho_{c y l} \cdot d V_{c y l}$ assuming a constant density $\rho_{c y l}$ within the cylinder volume $V_{c y l}$.

- Coordinate transformation into cylinder coordinates with $d V_{c y l}=r \cdot d r d \phi d z$ and a rotational center at

$$
\left(\begin{array}{l}
x_{c} \\
y_{c} \\
z_{c}
\end{array}\right)=\left(\begin{array}{c}
0 \\
0 \\
-\frac{s_{p i s t}}{2}
\end{array}\right)
$$

- Volume integrals are transformed with new boundaries<smiles>CC(C)CCOP(O)O</smiles>

- Yielding trigonometric integral parts are simplified

$$
\begin{aligned}
& \int \sin ^{2} \phi d \phi=-\frac{1}{2} \cdot(\sin \phi \cdot \cos \phi-\phi) \\
& \int \cos ^{2} \phi d \phi=\frac{1}{2} \cdot(\sin \phi \cdot \cos \phi+\phi)
\end{aligned}
$$

- Cylinder density is rewritten as

$$
\rho_{c y l}=\frac{m_{c y l}}{V_{c y l}}=\frac{m_{c y l}}{s_{p i s t} \cdot \frac{\pi}{4} \cdot\left(D_{b o r e}\right)^{2}}
$$

- Finally, a mass-independent moment of inertia is calculated via mass normalization for all directions dir

$$
j_{d i r}=\frac{J_{d i r}}{m_{c y l}}
$$

Thus, the formulations from equations (30) to (32) are finally mass-specific expressions $\left(\mathrm{m}^{2}\right)$ as in equations (8) and (9). Mathematical derivation yields $j_{x}=j_{y}$ and is therefore defined $j_{x y}$. 